\title{
CONF-8808/41-.9
}

\section{MEASURING THE MOMENTUM DISTRIBUTION OF THE UNPAIRED SPIN ELECTRONS IN FERROMAGNETS USING SYNCHROTRON RADIATION*}

\author{
Dennis M. Mills \\ Advanced Photon Source, Argonne National Laboratory \\ Argonne, IL 60439 USA
}

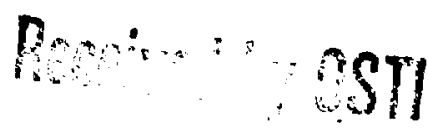

December 1988

CONF-8808141--9

JAN 2033

\begin{abstract}
The submitted manuxcript has been withored
by conerector of the U.S. Government oy contrector of the W.31-100ENG.38.

under contract No. M-31-109ENG.38.

Accordingty, tho U.S. Gowernment remins

nanexelusive, rovelty-fres licens ro publish

or reproduce the publithed form of this

contribution, or tlow oihers

U. S. Gewerment purpoins.
\end{abstract}

DE89 005864

Paper submitted to the proceedings of the 3rd International Conference on Synohrotron Radiation Instrumentation: SRI-88, August 29-September 2, 1988 , Tsukuba, Japan.

\section{DISCLAIMER}

This report was prepared as an acoount of work sponsored by an agency of the United States Government. Neither the United States Government nor any ugency thereof, nor any of their employees, makes any warranty, express or implied, or asumes any lesal liability or reaponsibility for the accuracy, ccmpleteness, or usefulneas of any information, apparatus, product, or process diaclowed, or represents that its use would not infringe privately owned rights. Reference herein to any specific commercial product, process, or service by trade name, trademark, manufacturer, or otherwise does not necescarily constitute or imply its endorsement, recommendation, or favoring by the United States Government or any ayency thereof. The views and opinions of authors expreased herein do not necessarily state or reflect those of the United States Gowernment or any agency thereof.

FThis work supported by the U.S. Department of Energy, BES-Materials Sciences, under contract no. W-31-109-ENG-38. 
MEASURING THE MOMENTUM DISTRIBUTION OF THE UNPAIRED SPIN ELECTRONS IN FERROMAGNETS USING SYNCHROTRON RADIATION

Dennis M. Mills

Advanced Photon Source, Argonne National Laboratory Argonne, IL 60439

\section{ABSTRACT}

The dominant term in the $x$-ray Compton cross-section of an electron. is the interaction of the photon and the electron's charge. Platzman and Tsoar many years. ago pointed out that there is also an interaction between an $x$-ray and the electron's spin and in principle this interaction can give information on the momentum distribution of the unpaired spin electrons in the solid. Unfortunately the spin sensitive term is not only small compared to the charge term, but in addition couples to the photons in first order only with that components of the $x$-ray beam that is circularly polarized. A lack of intense sources of circularly polarized $x$-rays combined with the relative small size of the spin sensitive term makes measurements of the momentum distributions of unpaired spin electrons difficult resulting in little experimental progress initlally made in spin or magnetic Compton scattering.

In the past several years interest in spin sensitive Compton scattering has been revived due in large part to the availability of intense beams of high energy photons from synchrotron radiation sources. The radiation from storage $r i n g$ sources has well def 1 ned polarization states; highly linearly polarized in the orbital plane and elliptically polarized above and below the plane of the orbit of the circulating particles. The high flux and unique polarization properties of synchrotron radiation sources have greatly facilitated measurements of the momentum distributions of the unpaired spin electrons in ferromagnetic solids. Recent results of the work of several groups will be presented along with some thoughts on the impact that the next generation of storage rings, such as the Advanced Photon Source, and insertion devices specifically designed to produce circularly polarized $x$-ray beams will have on the field of magnetic Compton scattering.

*This work supported by the U.S. Department of Energy, BES-Materials Science, under contract no. W-31-109-ENG-38. 
For over fifty years Compton scattering has been used as a tool in the study of electronic momentum distributions in condensed systems. After the initial work by Dumond and Kirkpatrick in the 1930's, however, there were few significant advances in the experimental technique until the advent of the high resolution solid state detector (SSD) in the $1960^{\prime} \mathrm{s}$. SSD's permitted the use of high energy (although low flux) radioactive gamma sources which markedly improved the quality of the data and extended the range of sample materials that could be studied. In the last decade work has proceeded to develop the necessary apparatus to extend Compton scattering experiments from investigating the momentum distribution of all the electrons to selectively looking at only the unpaired spin electrons. To progress further researchers need the magnetic Compton scattering equivalent of the SSD. It is my opinion that the new generation of high energy synchrotron radiation sources to be built will provide that impetus. The copious quantities of highly (linearly and circularly) polarized, collimated, short wavelength $x$-rays these storage $r i n g$ sources can provide are precisely what is needed for progress in this area.

\section{THEORETICAL BACKGROUND}

When an incident beam of monochromatic $x$-rays strikes an atom, some of the photon's energy can be transferred to an electron. If this electron were at rest, the energy spread of these inelastically scattered photons would depend önly on the energy width of the incident beam. In any real material, however, the electron will not be a rest and the energy spread of the Compton scattered photons will be Doppler broadened by the electron's velocity. Hence the line shape of the inelastically scattered photons, the so-called Compton profile, contains information on the velocity or momentum distribution of the electrons in the sample. (A more detailed analysis of the relationship between the shape of the Compton profile and the momentum distribution of the electrons and the approximations under which this relationship holds can be found in several recent articles. ${ }^{1,2}$ ) Utilizing the Born approximation, the differential cross-section for scattering of $x$-rays from a single electron can be written as ${ }^{3}$ :

$$
\frac{d^{2} \sigma}{d \Omega d \omega}=r_{0}^{2}\left(\frac{w_{2}}{w_{1}}\right) s(\underline{k}, \omega)
$$

where $r_{0}$ is the classical radius of the electron, $\omega_{1}\left(\omega_{2}\right)$, the incident (scattered) $x$-ray frequency, $\omega=\omega_{1}-\omega_{2}$, and $S(\underline{k}, \omega)$ is the generalized scattering factor. $S(\underline{k}, \omega)$ can be expressed as:

$$
s(\underline{k}, \omega)=\sum_{m, n}\left|M_{m n}\right|^{2} \delta\left(E_{n}-E_{m}-\omega\right)
$$

where $M_{\mathrm{mn}}$ are the matr $1 \mathrm{x}$ elements $\left\langle\mathrm{m}\left|\mathrm{e}^{1 \underline{\mathrm{k}} \cdot \underline{\mathrm{r}}}\right| \mathrm{n}\right\rangle$. In 1970 Platzman and Tsoar ${ }^{4}$ showed that when relativistic effects are taken into account, $M_{m n}$ can be written (to order $h w / m c^{2}$ ) as

$$
M_{m n}=A \delta_{m n}+i\left(\frac{\hbar \omega}{m c^{2}}\right) B \cdot \underline{\sigma}_{m n}
$$




$$
\begin{aligned}
& A=\left(\underline{\varepsilon}_{1} \cdot \underline{\varepsilon}_{2}\right), \\
& \left.\underline{B}=-\left(\underline{\varepsilon}_{1} \cdot \underline{\varepsilon}_{2}\left(\hat{k}_{1} \times \hat{k}_{2}\right)-\frac{1}{2}(\hat{g} \cdot \hat{g})\left(\underline{\varepsilon}_{1} \times \underline{\varepsilon}_{2}\right)-\hat{g} \times\left(\hat{g} \times \underline{\varepsilon}_{1} \times \underline{\varepsilon}_{2}\right)\right) ; \hat{g}=\hat{k}_{1}-\hat{k}_{2}\right)
\end{aligned}
$$

and $\sigma$ is the electron spin. The first term yields the standard Thompson cross-section and the second leads to the spin-dependent effects. Two things are immediately apparent: (1) the magnetic or spin term is scaled relative to the charge term by the factor $\left(h \omega_{1} / \mathrm{mc}^{2}\right.$ ) and (2) the terms are out of phase by $90^{\circ}$. The physical origin of these factors comes about due to the different interactions that are involved in the electronic and the spin scattering. When eqn. $3 a$ is squared to produce the cifferential cross-section; the cross term, i.e. the term linear in the spin, will vanish unless $A$ has an imaginary component. In this single electron model, this occurs only if the polarizations are complex; namely circularly polarized $x$-rays are required to pick up the leading magnetic scattering term. It should be noted that the pure magnetic term, the term proportional to $\left(B^{\circ} \sigma\right)^{2}$, does not require circularly polarized $x$-rays to be observed. However, as shall be seen later this term is very small compared to the electronic scattering intensity and is not amenable to the technique that will be employed to isolate the spin from the charge scattering since it is an even function of the spin.

The explicit form for the differential cross-section, to first order in the spin, is given below following the notation of Lipps and Tolhoek. ${ }^{5}$

$$
\begin{aligned}
& \frac{d^{2} \sigma}{d \Omega d p_{z}}=\frac{r_{0}^{2}}{2}\left(\frac{k_{2}}{k_{1}}\right)\left[\left(\Phi_{0}+P_{\ell} \Phi_{l}\right) J^{+}\left(P_{z}\right)+P_{c} \Phi_{c}(\underline{\sigma}) J^{-}\left(P_{z}\right)\right] \\
& \Phi_{0}=\left(1+\cos ^{2} \phi\right)+\left(k_{1}-k_{2}\right) \frac{h_{c}}{m_{0} c^{2}}(1-\cos \phi) \\
& \Phi_{\ell}=\sin ^{2} \phi \\
& \Phi_{c}(\underline{g})=-(1-\cos \phi) \underline{\sigma} \cdot\left(\underline{k}_{1} \cos \phi+\underline{k}_{2}\right) \frac{\hbar_{c}}{m_{0} c^{2}}
\end{aligned}
$$

where $k_{1}\left(k_{2}\right)$ is the incident (scattered) wavevector, $P_{\ell}\left(P_{c}\right)$ the linear (circular) degree of polarlzation and $\phi$ the scatteringlangle. The information on the momentum distribution are contained in the $\mathrm{J}^{ \pm}\left(P_{z}\right)$ :

$$
J^{ \pm}\left(P_{z}\right)=\iint \sum_{\text {bands }}[n+(p) \pm n+(p)] d p_{x} d p_{y}
$$

where $n+(p)(n+(p))$ is the spin-up (spindown) momentum distribution. As can be seen from the above equations, with a typical incident $x$-ray energy of tens of kilovolts, the ratio of the spin-to-charge Compton intensities is only a few percent. This problem is further compounded in an atom where essentially all the electrons contribute to the charge scattering but only those unpaired spin electrons participate in the magnetic scattering. Hence, even in Fe, which is a relatively low $z$ material with a high moment per atom $\left(2.2 \mu_{B}\right)$, the spin component of the 
Compton profile is less than one percent of the entire profile. From an experimental viewpoint then, one of the major challenges in measuring spin dependent Compton profiles is the problem of extracting it from the much larger electronic component. The most common technique in use is that of a spin flip method. Data is taken first with the spins aligned in one direction (spin-up) and then reversed (spin-down). From equations 4 it can be seen that the spin flip will have no effect on the $\Phi_{0}$ and $\Phi_{\ell}$ but will change the sign of the spin dependent term. Subtraction of spin-up and spin-down data will then eliminate the charge contribution leaving a remainder proportional to the differences in the spin-up and spin-down momentum distributions. This spin flipping is achieved by an application of an external magnetic field on the sample.

Note that a sign reversal can also be produced in the spin dependent term if the handedness of circularly polarized radiation is changed. This tecinique would also serve as a means to isolate the spin dependent Compton profile and might be particularly useful for those materials where it is difficult to reverse the direction of the moment with external magnetic fields. One can also get the individual Compton profiles from subtracting data taken in a spin-up (or spin-down) configuration from a pure electronic Compton profile of the same material. (A pure electronic Compton proflle can be obtained by orienting the sample so that the moment is perpendicular to the scattering plane for example.) Regardless of the technique employed, the key to success of these methods is large quantitites of circularly polarized $x$-rays.

\section{SOURCES OF CIRCULARL $\ddot{Y}$ POLARIZED X-RAYS}

The pioneering experimental measurements of spin dependent momentum distributions were performed using nuclear oriented radioactive sources. When sufficiently cooled and placed in an external magnetic field, it is possible to enhance the probability of those nuclear transitions that emit circularly polarized gamma rays. Starting initially with $57 \mathrm{Co}$ sources (122kev) and later switching to ${ }^{191}$ Os (129 kev) sources, Sakai and coworkers 7,8 have achieved a degree of circular polarization of 0.80 and fluxes of about $10^{6}$ photons $/ \mathrm{cm}^{2}$ onto the sample; the count rate being limited by self heating effects of the radioactive source. With these count rates data collection times of several hundred hours are necessary in order to collect one magnetic Compton profile.

Needless to say, these studies were extraordinarily difficult and progress in the area of experimental determined spin dependent Compton profiles was slow. Clearly if any major inroads were to be made, new sources of high intensity, circularly polarized x-rays were required. Fortunately $x$-rays generated from high energy storage $r$ ings have been able to supply that increased flux. The possibility of exploiting the unique properties of synchrotron radiation for use in magnetic compton scattering studies is still in its infancy. Interestingly enough two different methods of obtaining clrcularly polarized radiation has already been successfully employed to obtain magnetic Compton profiles with statistical accuracies comparable to (or better) that the original data taken with gamma ray sources but with measurement times an order of magnitude less! Figure 1 shows the polarization properties of the radiation emitted from a dipole magnet. On axis, that is in the plane of the orbiting particle, the radiation intensity is a maximum and the polarization is nearly coinpletely linear. As one goes above (below) the orbital plane, the radiation becomes increasingly right hand (left hand) circular polarized; but with a loss of flux as compared to the on-axis intensity. Cooper and his colleagues $9,10,93$ working at the synchrotron 


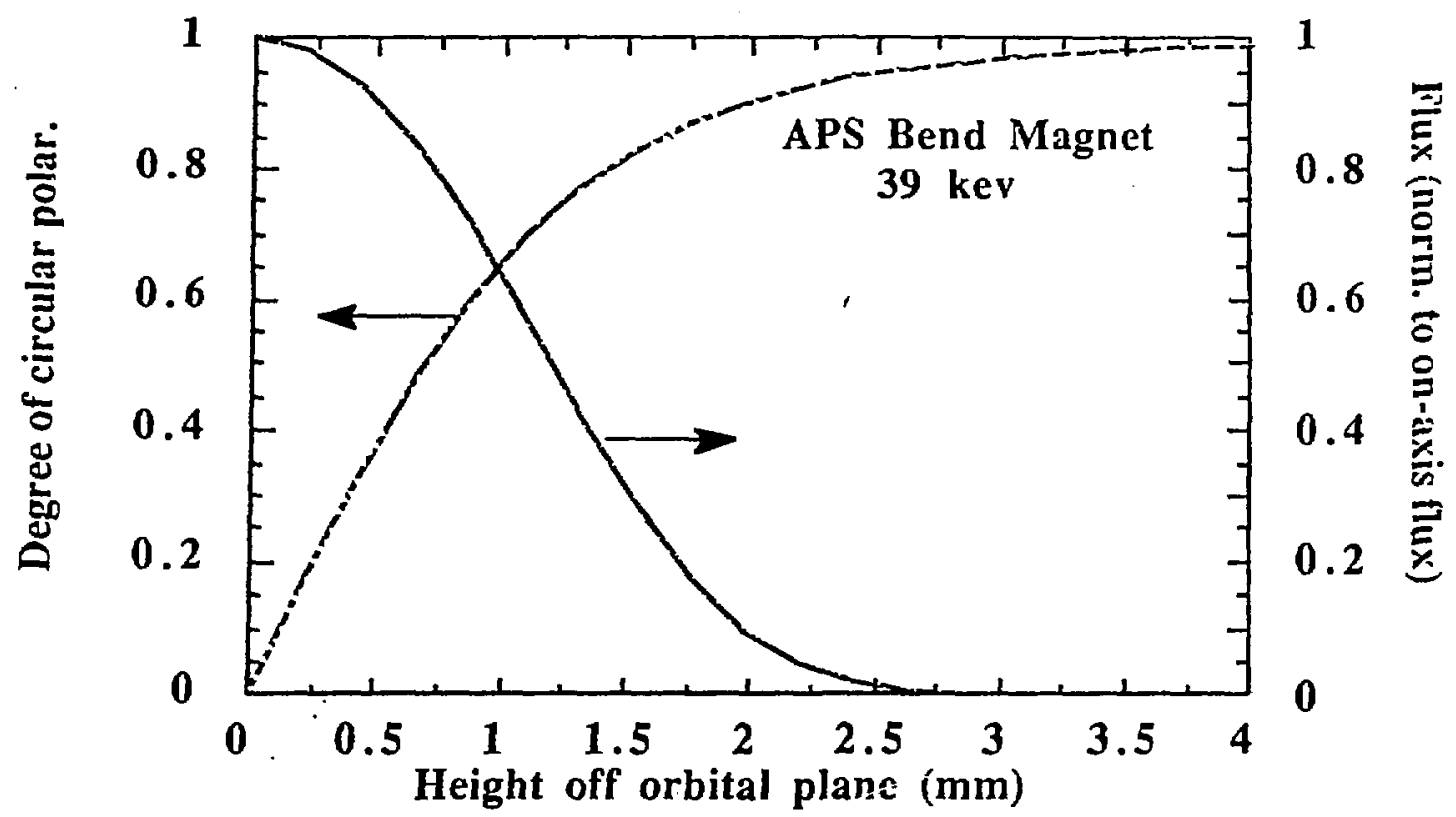

Fig. 1. Degree of circular polarization is plotted as a function of helght above (or below) the crbltal plane 30 meters from an APS band magnet. Also plotted is the height dependence of the flux normalized to the on-axis flux valve. This has been calculated at $39 \mathrm{keV}$, twice the critical energy of the bend magnet radiation. The effects of finite emittance and slit size have been neglected in these calculations.

Radiation Source (SRS) at Daresbury, UK have exploited this property of the radiation by arranging their apparatus to see only radiation above (or below) the orbital plane. Using this "inclined view" geometry they have collected data on several transitions metal ferromagnets and recently collected the first directional spin dependent Compton profiles. (See section on EXPERIMENTAL DATA for further details). With the SRS running at $2.5 \mathrm{Gev}$ and 100 ma they obiained, from a high fleld superconducting wavelength sifter, an intensity at the sample of $10^{9}$ photons $/ \mathrm{cm}^{2}$ with the degree of circular polarization about 0.80 at 60 kev.

The standard technique for increasing photon flux at storage $r$ ing sources is through the introduction of insertion devices; wigglers or undulators. Insertion devices are magnetic structures with alternating magnetic field directions perpendicular to the orbital plane of the part1cle. As the particle travels through the insertion device it is forced to oscillate back and forth (The magnetic flelds are carefully trimmed so that the particles net deflection after going thought the device is zero.) In the case of a wiggler, one can think of each pole as a source point of radiation, so that if a device has $N$ poles, the photon flux is $N$ times that of a single dipole source point. Unfortunately these alternating fields, the very origin of the increased flux, destroy the circular polarization above and below the orbital plane. Because they produce alternately righthand and lefthand polarization above the orbital plane, the resulting of $f$-axis radiation f'rom wigglers is almost totally unpolarized. However, one can make use of the ample quantity of linear polarized $x$-rays by conversion of the IInearly polarized,radiation to circular polarized radiation via x-ray phase plates. Mills has successfully produced circularly polarized $x$-rays at $40 \mathrm{kev}$ with a degree of circular 
polar!zation between 0.40 and 0.70 with an $x$-ray phase plate and used these $x$-rays to collect spin dependent Compton profiles of the transition ferromagnets ( $\mathrm{Fe}, \mathrm{Co}$, and $\mathrm{Ni}$ ) and $\mathrm{Gd} .{ }^{12}$ This data was collected at the Cornell High Energy Synchrotron Source (CHESS) on a dipole source ( 5.5 $G \in V$ and $25 \mathrm{ma}$ ) with an incident beam intensity of greater than $10^{9}$ photons $/ \mathrm{cm}^{2}$.

\section{EXPERIMENTAL SET-UP}

The general experimental set-up is shown in Figure 2. As can be seen from equations 4 for a given incident $x$-ray energy, the spin contribution to the cross-section is maximized in a back scattering geometry with the spin direction parallel to the incident beam. (See Fig. 3) An electro-magnet is used to align and reverse the spins in the sample. Depinding on the details of the experiment, either a reflection or transmission geometry can be used.

To date all magnetic Compton scattering profiles have been collected with energy dispersive solid state detectors due to the dearth of magnetically scattered photons. This has necessarily resulted in experimental data being of 'modest' momentum resolution; typically 0.7 to $0.9 \mathrm{a} . \mathrm{u}$. (Recall that Compton profiles are generally only several au's wide!). With current detector technology, improvements in momentum resolution will be difficult to achieve unless large increases in the flux of circularly polarized $x$-rays can be made avallable so that SSD's can be replaced with energy dispersive crystal analyzers.

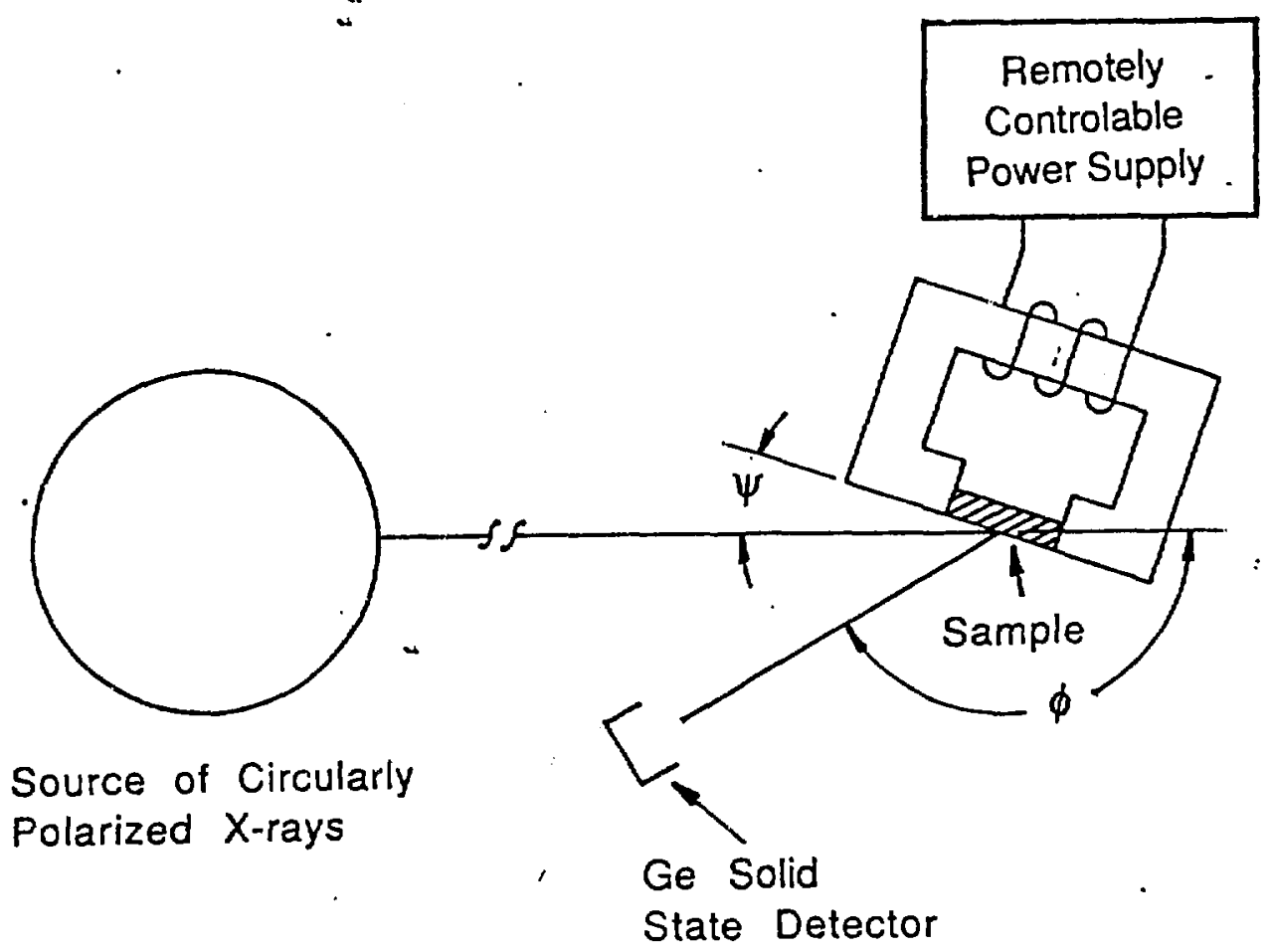

Fig. 2 Schematic of the experimental layout for magnetic Comption scattering. Shown here is a reflectance geometry (entrance and exit beam on same side of sample); however, in some instances a transmission geometry (entrance and exit beams on different sides of the sample) is more advantageous. 


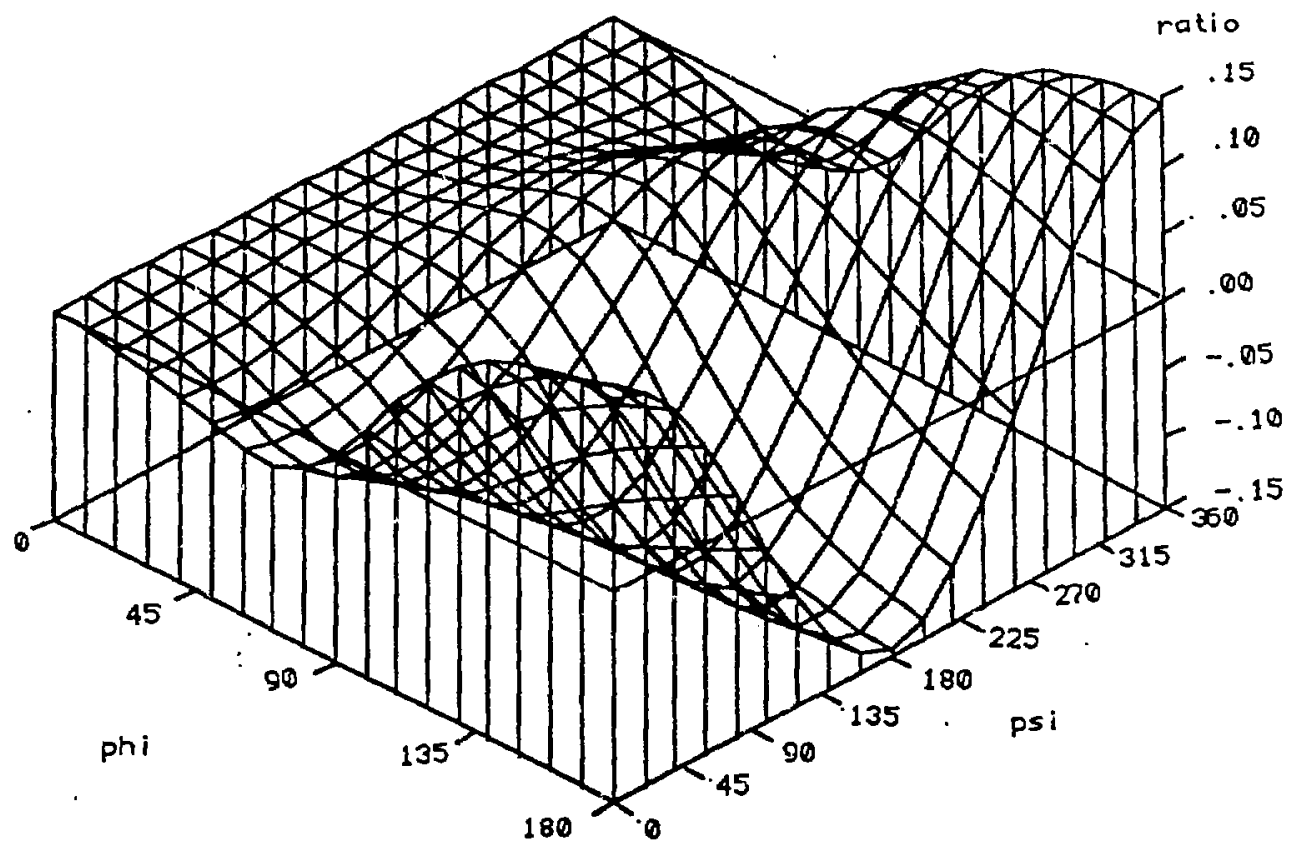

Fig. 3. The magnetic-ta-electronic cross section ratio for a single electron plotted as a function of scattering angle $\phi$ for several different s.pin orientations $\psi$. The spin is assumed to lie in the scattering plane, $\psi=0$ is along the incldent beam direction. These were calculated for an incident $x$-ray energy of 40 keV. The ratio scales directly with the photon energy.

\section{EXPERIMENTAL DATA}

Because of the experimental difficulties involved, to date magnetic Compton scattering experiments have been 1.mited systems of relatively high magnetig moments per atom ang $1 \%$ atomic numbers, $1 . e$. $\mathrm{Fe} 7,8,9,12,13$, Co $12,: 3, \mathrm{Ni} 12$ and $\mathrm{MnFe}_{2} \mathrm{O}_{4}$, with the single exception being gadolinium. A representative sampling of that data is shown in Figs. 4, 5 and 6 . All data from the pure ferromagnetic transition metals show a dip in the momentum distribution about $\mathrm{p}_{z}=0$; a manifestation of the relative abundance of the negative spin density far from the atomic core location. This dip has been also been predicted from theoretical studies 14,21 and the source of this reverse magnetization has been associated with the sp electrons. In the calculations for $F e$, where a majority of the theoretical work has been carried out, the negative spin components is gonsistently underestimated. Some recent work by cooper and colleagues 10 have indicated where the discrepancy between the measurements and the calculations may lie. They have recorded, for the first time, directional Compton profiles and have been able to compare them directly with calculations, identifying the crystal orientations where the major differences appear. (See Eig. 6).

In an attempt to contrast the difference in the profiles between intinerate and localized spin electrons, Sakai and Sekizawa have measured the spin dependent Compton profiles of $\mathrm{Fe}$ and ferrimagnetic $\mathrm{Mn}$ ferrite. Contrary to what might be expected in a ferrimagnetic insulator, they found that Mn ferrite also displayed the characteristic dip about $p_{z}=0$ normally associated the the itinerate nature of the unpaired spin electrons. They have conjectured that this may be due to different momentum distributions of the $3 \mathrm{p}$ and $4 \mathrm{~s}$ electrons between $\mathrm{Mn}^{+2}$ and $\mathrm{Ee}^{+3}$. 

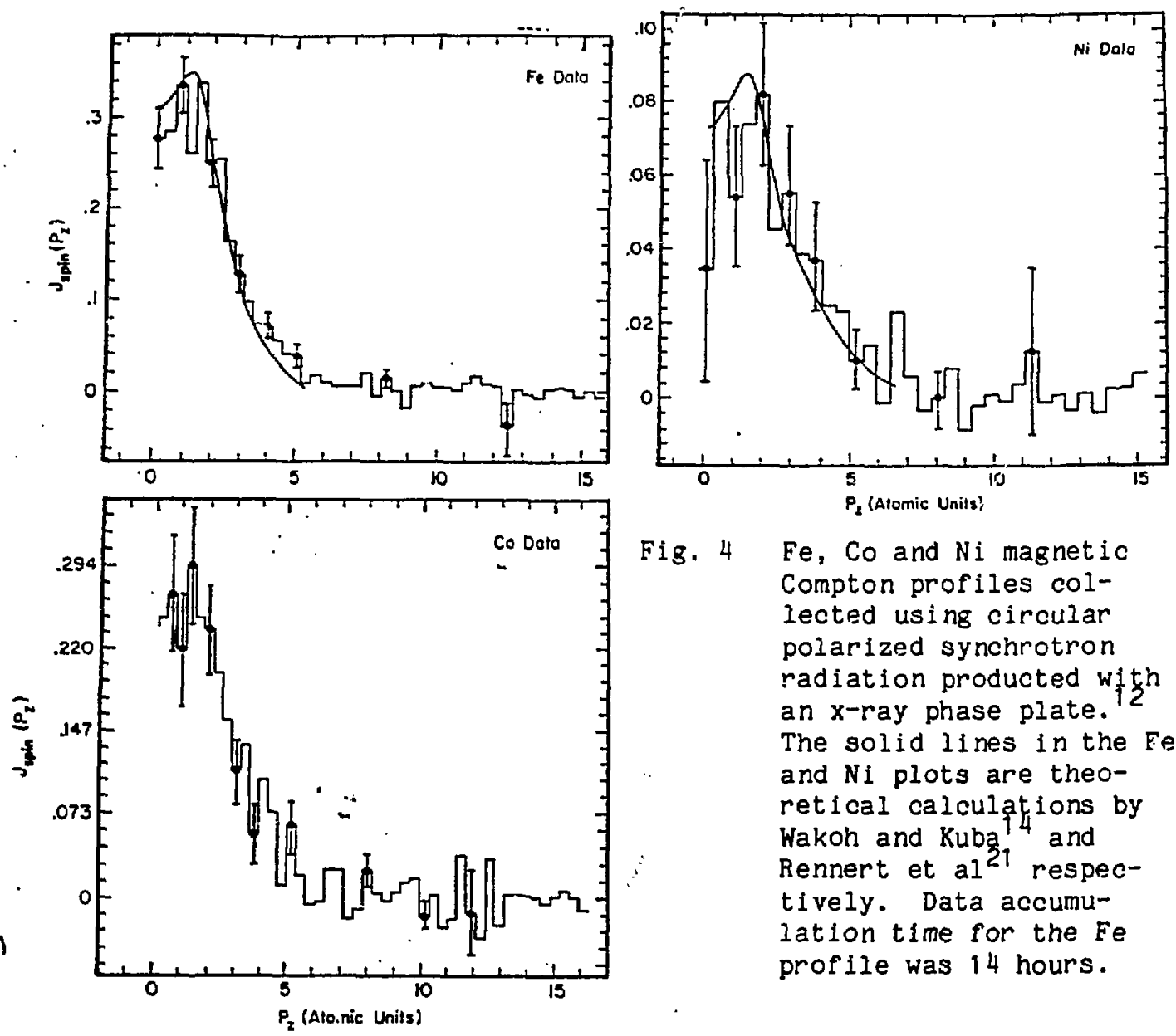

Fig. $4 \mathrm{Fe}$, Co and $\mathrm{Ni}$ magnetic Compton profiles collected using circular polarized synchrotron radiation producted with an $x$-ray phase plate. The solid lines in the $\mathrm{Fe}$ and $\mathrm{Ni}$ plots are theoretical calculations by Wakoh and Kubat and Rennert et al ${ }^{21}$ respectively. Data accumulation time for the $\mathrm{Fe}$ profile was 14 hours.

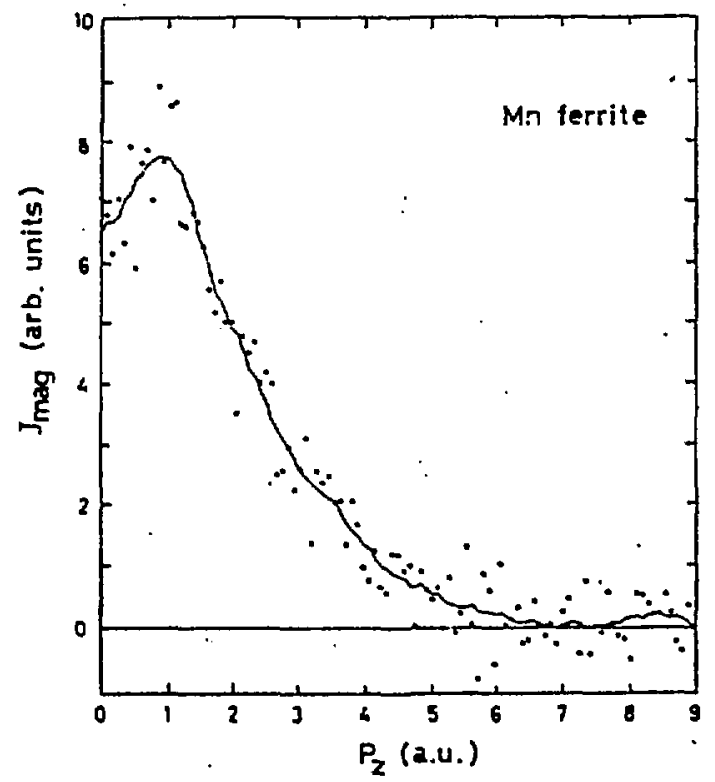

F1B. 5 Magnetic Compton profile of $\mathrm{MnFe}_{2} \mathrm{O}_{4}$ gollected using a radioactive

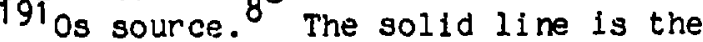
experimental data after a smoothing routine had been applied. Data accumulation time was 333 hours. 


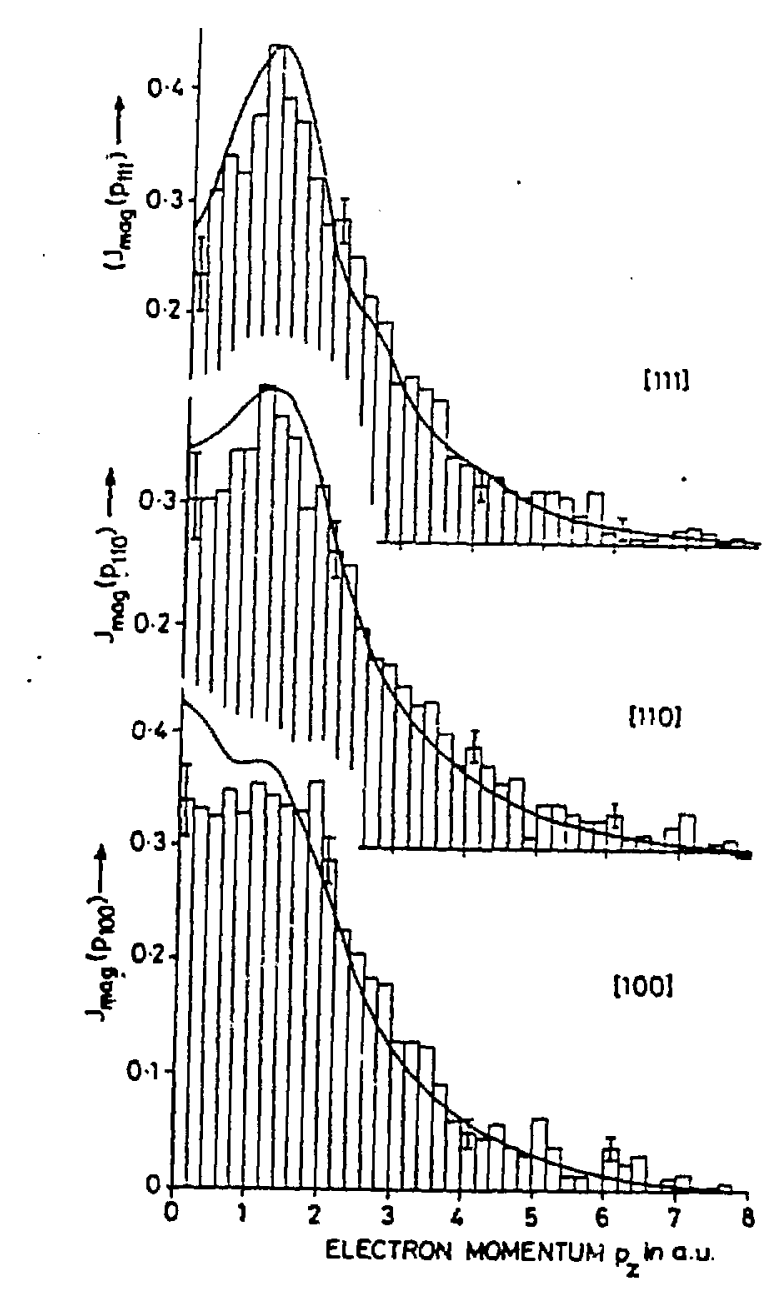

Fig. 6 Directional magnetic Compton proflies for $F e$ taken with off-axis synchrotron radiation. The solid line is an APW calculation ${ }^{10}$ convoluted with the experimental resolution function. Data collection time for each profile as about 20 hours.

Preliminary investigations into the spin Compton profile of Gd, rare earth ferromagnet with atomic-like magnetism, has indicated that 6,12 the dip about the origin is absent in this materlal; showing infact that all spin-denendent Compton proflles don't look alike.

\section{FUTURE DIRECTIONS}

Unless more potent sources of circularly polarlzed $x$-rays become avaliaule so that detalled and systematic studies of materials can be undertaken, magnetic Compton scattering experiments will soon be relegated to that class of experiments which I call proof of principle; 1.e. interesting from a experimental viewpoint but of little use to the better understanding of the physical properties of the sample. I believe 
that storage $r i n g$ sources can provide that Increase in flux required to take tinis technique from a proof of principle status to a useful and incisive tool for the siudy of the momentum distributions of the unpaired spin electrons in condensed matter systems. The next generation of synchrotron radiation sources, the Advanced Photon Source (APS) to be bullt at Argonne and the European Synchrotron Radiation Facility (ESRF) beling built at Grenoble, are high energy storage rirgs (6-7 Gev) that have been specifically designed for use with insertion devices. Both these facts are important to Compton scattering researchers; the high beam energy meaning increased flux at shorter $x$-ray wavelengths and the compatibility with insertion devices meaning the possibility of tailoring the emitted radiation to the specific needs of the experimenter. Many advances are being made in the area of exotic insertion devices designed explicitly for the production of circularly polarized x-rays. Hellical 15 undulators, crossed planar undulators, ${ }^{16}$ crossed-retarded field undulators and wigglers ${ }^{17}$ and asyminetric wigglers ${ }^{i 8}$ have all been proposed for the production of intense beams of circularly polarized x-radiation. In the very near future a distorted helix wiggler is to go on the $6 \mathrm{Gev}$ accumulator ring, TRISTAN, at KEK in Japan for the production of high fluxes of elliptically polarized $x$-rays at energies between 40 and $60^{19}$ kev. We excitedly await the the results from this new insertion device design.

The construction of these new storage $r i n g$ sources has precipitated a renewed interest in $x$-ray optics; in particular short wavelength $x$-ray optical components. Many of these novel components can be installed at currently operating 'synchrotron radiation sources. For instance, only recently has work been done on $x$-ray phase plates that operate at energies above $40 \mathrm{kev}$ and this study was one a dipole magnet source winich had a critical energy (half power point) of $-10 \mathrm{kev}$. The transfer of the same device to the wiggler beamline at CHESS should afford a 50 fold increase in intensity due to the higher critical energy (29 kev) of the wiggler. (One of the experimental obstacles of working on these high field insertion devices is the handling of the power they put out, often many kilowatts!) Focusing optics can produce considerable increases in flux on the sample over that of the flat double crystal monochrometers available on most $x$-ray beamlines. Here the problem is one of produeing efficient focusing optical components capable of working at $40 \mathrm{kev}$ and above. An optimized match of the band-pass of the monochromator to the resolution of the detector can significantly improve the quality of magnetic Compton scattering profiles. Given the current energy resolution of solid state detectors (several hundred ev in the energy region of interest) experimentai momentum resolution is not compromised if the bandpass of the monochromated radiation is increased from the current value of several ev's (typical of perfect single crystal monochromators) to that approaching the detector resolution. In this fashion an order of magnitude increase in flux on the sample realizeable with virtually no effect on the resultant momentum resolution. To i ncrease the moment um resolution over what one can obtain with a SSD, dispersive analyzing crystals are required. Analyzing crystals used in a transmission mode in conjunction with a $1 D$ detector (film, image plates, wire detactors, CCD's) can provide momentum resolutions to better that 0.1 au but at an exorbitant cost in counting rates. The effestive operation of $x$-ray phase plates, focusing optics, wide band-pass monochromators and analyzing crystals (or perhaps even combinations of these devices) at short $x$-ray wavelengths presents a stimulating challenge to designers of moder $n x$-ray optical components. Concurrent with improvements in the area of $x$-ray production and optical components, advances in semiconductor microrabrication techniques are allowing detectors to be produced with 
resolutions 10 times better that currently available SSD's. 20 The con verge of all these technologies, insertion device development, $x$-ray optical components, and detector development in the next several years will most certainly produce new and thought providing studies in the field of spin-dependent momentum distribution measurements.

\section{ACKNOWLEDGEMENTS}

The author would like to thank both Profs. Sakai and Cooper for their discussions and insights into the field of spin dependent magnetic Compton scattering. The author would also like to thank the organizers of the Workshop on Momentum Distributions for inviting him to participate in their meeting. Support for this work was provided by the Department of Energy under contract number No. W-31-109-ENG-38.

\section{REFERENCES}

1. "Compton Scattering," B. W. William, ed., McGraw Hill, New York 1977.

2. Cooper, M., Rep. Prog. Phys., 48 p. 415, 1985.

3. Elsenberger, P. and Platzman, P., Phys. Rev. A, 2, p. 415, 1970.

4. Platzman, P. and Tsoar, N., Phys. Rev. B, 2, p. 355G, 1970.

5. Lipps, F. W. and Tolhoek, H. A., Physica $x \bar{x}$, p. 395, 1954.

6. M. Cooper, private communication.

7. Sakai, N. and Ono, K., Phys. Rev. Lett3. 37, p. 351, 1976.

8. Sakal, N. and Sekizawa, H., Phys. ReV. B 36, P. 2164, 1987.

9. Cooper, M. J., Laundy, D., Cardwell, D. A., Timms, D. N., Holt,. R. S. and Clark, G., Phys. Rev. B 34, p. 5984, 1986.

10. Cooper, M. J., Collins, S. P., Timms, D. N., Brahmia, A., Kane, P. P., Holt, R. S. and Laundy, D., Nature, 333, p. 151, 1988.

11. Mills, D. M., Nuc. Inst. and Meths. A226, p.531, 1988.

12. Mills, D. M., Phys, Rev. B. 36, p. $6 \overline{178}, 1987$.

13. Timms, D. N., Brahmia, A., Collins, P., Collins, S. P., Cooper, M. J., Holt, R. S., Kane, P. P., Clark, G. and Laundy, D., J. Phys. F, 18 , p. L57, 1988.

14. Wakoh, S. and Kubo, Y., Jour. of Mag. and Mag. Matls. , p. 202, 1977.

15. Kincaid, B. M., J. Appl. Phys. 48 p. 2684, 1977.

16. Kim, K. J., Nuc. Inst. and Methods, A219, p. 425, 1986.

17. Onuki, H., Nuc. Inst. and Methods. A2 $\overline{46}$, p. $94,1986$.

18. Goulon, J. Elleaume, P. and Raoux, D., Nuc. Inst. and Methods, A254, p. $192,1987$.

19. Yamamoto, S. Shiga, T., Sasaki, S., and Kitamura, H., to be published in Rev. Scl. Inst.

20. McCammon, D., Juda, M. Zhang, J., Holt, S. S., Kelly, R. L., Mosley, S. H. and Szymkowiak, A. E., Jap. Journ. of Applied Shys., 26, Suppl. 26-3 (1987).

21. Rennert, P., Carl, G. and Hergert, W., Phys. Stat. Sol. (b) 120, p. $273,1983$. 